\title{
Barreras, facilitadores y motivos de la práctica deportiva de deportistas con discapacidad intelectual
}

\author{
Jorge ABELLÁN ${ }^{1}$ y Nuno JANUÁRIO ${ }^{2}$ \\ ${ }^{1}$ Universidad de Castilla-La Mancha. España \\ ${ }^{2}$ Universidade de Lisboa. Portugal
}

(Recibido, 13 de Abril 2017; Aceptado, 22 Junio 2017)

RESUMEN: El objetivo del presente trabajo es identificar las barreras, facilitadores y motivos para la práctica de actividad física y deporte en las personas con discapacidad intelectual, considerando el sexo, la carga de entrenamiento y la ocupación de los participantes. En el estudio han participado 23 deportistas con discapacidad intelectual, a los que se ha aplicado un cuestionario en forma de entrevista. Se ha utilizado estadística descriptiva y pruebas no-paramétricas para la comparación de grupos. Los resultados indican que las principales barreras están relacionadas con el tiempo de desplazamiento al lugar de entrenamiento; los principales facilitadores están relacionados con el apoyo del entorno del deportista; los principales motivos para la práctica están relacionados con mejorar la salud y con factores sociales. Considerando las variables independientes se han encontrado algunas diferencias estadísticamente significativas. Los hombres perciben que ducharse en las instalaciones es una barrera; los deportistas con más carga de entrenamiento perciben el desplazamiento como una barrera; y el apoyo de los padres es un facilitador para los deportistas que trabajan. En base a los resultados se presentan implicaciones a nivel teórico y a nivel práctico como ayuda para mejorar la práctica deportiva en las personas con discapacidad intelectual.

Palabras clave: Deporte adaptado, discapacidad intelectual, inclusión.

\section{Barriers, facilitators and motives of sport participation for athletes with intellectual impairments}

ABSTRACT: The objective of the present study is to identify the barriers, facilitators and reasons for practicing physical activity and sport in people with intellectual disabilities, considering the gender, the training load and the occupation of the participants. The study involved 23 athletes with intellectual impairments, to whom a questionnaire has been applied in the form of an interview. Descriptive statistics and non-parametric tests for group comparison have been used. The results indicate that the main barriers are related to the time of displacement to the training place; the main facilitators are related to the support of the athlete's environment; the main reasons for the practice are related to improving health and social factors. Considering the independent variables, we found some statistically significant differences. Men perceive that showering on the lockers is a barrier; Athletes with more training load perceive displacement as a barrier; and the support of parents is a facilitator for working athletes. Based on the results, theoretical and practical implications are presented as help to improve the practice of sports in people with intellectual disabilities.

Keywords: Adapted sport, intellectual impairment, inclusion. 
Correspondencia: ${ }^{1}$ Jorge Abellán, Facultad de Educación, Universidad de Castilla-La Mancha. Campus universitario s/n. C.P. 16071, Cuenca (España). Email: jorge.abellan@uclm.es

\section{Introducción}

Se ha demostrado que las actividades físicas y deportivas son una buena herramienta para la socialización de las personas con discapacidad (Crawford, Hollingsworth, Morgan y Gray, 2008). Sin embargo la participación de dicha población en actividades de este tipo ha sido baja (Yazdani, Yee y Chung, 2013), tanto en educación física (Block y Obrusnikova, 2007), como en el contexto deportivo (Bult, Verschuren Jongmans, Lindeman y Ketelaar, 2011). El desarrollo de la investigación en el estudio de las barreras y los facilitadores de la práctica deportiva en personas con discapacidad intelectual (en adelante DI) es inexistente o muy escaso, de hecho han sido expresamente no incluidos en algunos de los estudios previos con el objetivo de explorar las diferentes barreras y facilitadores de los deportistas paralímpicos (Jaarsma, 2014).

A pesar de que no existen trabajos previos en la población objeto de estudio en este trabajo, las barreras y facilitadores de la práctica deportiva si han sido identificados en los deportistas paralímpicos y las personas con discapacidad. De manera general, las principales barreras que influyen en la práctica de deporte y actividad física por parte de las personas con discapacidad son: accesibilidad a los espacios públicos (Kirchner, Gerber y Smith, 2008), costes financieros, falta de compañeros y dependencia de otros (Jaarsma, Geertzen, de Jong, Dijkstra y Dekker, 2014), falta de formación de los profesionales (Brian y Haegele, 2014), falta de oportunidades consistentes (Haegele y Porretta, 2015), falta de seguridad (Greguol, Gobbing y Carraro, 2015) y poca información sobre oportunidades de práctica (Greguol et al.,2015; Perkins, Columna, Lieberman y Bailey, 2013). En cuanto a los factores identificados como facilitadores, las investigaciones previas han determinado que dichos factores son: la adquisición de habilidades motrices (Lieberman, Robinson y Rollheiser, 2006; Perkins et al., 2013), la mayor capacidad financiera del practicante y el sexo (para los hombres) (Ajuwon, Kelly y Wolffe., 2015), la motivación (Perkins et al., 2013), los niveles de actividad física de los padres (Greguol et al., 2015), la determinación personal (Kirchner et al., 2008), los contactos sociales, la diversión y la formación académica del practicante (Jaarsma, Geertzen et al., 2014) y el soporte familiar y de los profesionales (Greguol et al., 2015; Haegele y Porretta, 2015; Jaarsma, Geertzen et al., 2014; Perkins et al., 2013).

El desarrollo del deporte para personas con DI siempre ha sido menor que el del resto de las discapacidades, este pobre progreso del deporte para personas con DI, en comparación con otros colectivos con DI puede tener una raíz histórica, ya que la DI fue la última en incorporarse al movimiento paralímpico, además de estar fuera de dicho movimiento desde Sidney 2000 hasta Londres 2012, donde se ha incorporado con un número de pruebas y deportes mucho menor al resto (Abellán y Sáez-Gallego, 2014; Mendoza, 2009). Sin embargo si han existido estudios sobre la participación en actividades físicas y deportivas en personas con DI. Robertson y Emerson (2010) encontraron que el $41 \%$ de las personas con DI entrevistadas realizaron deporte en el último mes, el 34\% de los que no habían realizado declaró que le gustaría realizarlo. La participación en actividades deportivas en este trabajo se asoció a algunas características individuales y socioeconómicas, pero no a las necesidades específicas de apoyo. Además se han investigado las barreras de las personas con DI en la participación de actividades de ocio (en el (c) Psy, Soc, \& Educ, 2017, Vol. 9(3) 
que se incluía la práctica de actividades físicas). Los resultados extraídos del trabajo de Badia, Orgaz, Verdugo, Ullán y Martínez (2011) muestran que la participación en actividades de ocio están determinadas más por factores personales y por barreras percibidas que por factores relativos a la propia discapacidad. Entre las barreras percibidas referidas a la realización de actividades físicas en el tiempo libre destacan no ser demasiado bueno en la tarea a realizar y no tener a alguien que les enseñe a realizar la actividad.

La percepción de las barreras y los facilitadores, como también los motivos que llevan a los practicantes a adherirse a la práctica deportiva ha sido muchas veces apuntado como un factor determinante de la práctica deportiva en general (Januário, Colaço, Rosado, Ferreira y Gil, 2012), pudiendo todavía ser más relevante en las personas con DI. Los motivos de práctica deportiva han sido analizados recientemente en los deportistas paralímpicos españoles (Torralba, Braz y Rubio, 2017), los resultados indican que la participación de este colectivo está muy relacionado con factores personales y de superación, sin embargo, el grupo de deportistas paralímpicos con DI no ha sido incluido en la población de estudio.

Teniendo en cuenta lo expuesto anteriormente, el objetivo de este trabajo es sensibilizar a la población en general y, en particular, a los profesionales en el campo de la Educación Física y el Deporte, sobre las principales barreras y los facilitadores de los deportistas con DI. De una forma más concreta, se pretende responder a las siguientes cuestiones. ¿Cuáles son las principales barreras y los facilitadores a los que se enfrentan los deportistas con DI? ¿Influyen las características personales como la edad, el sexo y la ocupación en la percepción de las barreras y de los facilitadores por parte de los deportistas con DI? ¿Perciben los deportistas con una carga de entrenamiento más elevado las mismas barreras y facilitadores que los que entrenan menos?

\section{Método}

\section{Participantes}

Participaron 23 deportistas con DI (14 hombres y 9 mujeres) con una edad comprendida entre 18 y 59 años $(M=33.91, D T=10.10$ años) que tenían licencia deportiva en el momento de la recogida de los datos. Respecto a su ocupación diaria, el 13\% estaba en casa o en una institución, el $22 \%$ estudiaba, el $48 \%$ trabaja, el 13\% estaba en situación de desempleo y el restante $4 \%$ estaba jubilado. En cuanto a su participación deportiva, el 57\% practicaba natación, el $22 \%$ practicaba baloncesto, el $17 \%$ practicaba fútbol sala y el restante $4 \%$ realizaba atletismo, además cabe destacar que el $56 \%$ de los participantes entrenaba más de un deporte a la semana, combinando los anteriormente comentados.

El 39.13\% de los participantes entrenaba más de 3 horas a la semana, mientras que el restante $60.87 \%$ entraba 3 horas o menos a la semana.

\section{Instrumento}

Se ha utilizado un cuestionario basado en el modelo International Classification of Functioning, Disability and Health (ICF) (OMS, 2011) y en el trabajo previo de Jaarsma, Geertzen et al. (2014). Se ha adaptado al contexto español utilizando la versión en inglés y la versión en portugués, empleadas por Jaarsma (2014) y Damas, Januário, Rosado y Pereira 
(2016) respectivamente. A pesar de que todos los participantes sabían leer y escribir, y debido a las características específicas de su discapacidad, se utilizó el cuestionario como base para realizar una entrevista, de manera que todos los participantes recibieron ayuda a la hora de completarlo, por parte del equipo de investigación y de los entrenadores de los participantes.

El instrumento quedo finalmente construido por 23 ítems, divididos en 4 factores (desplazamiento, tiempo, apoyo e instalaciones) relacionados con las barreras y los facilitadores y 8 ítems, para los motivos para la práctica (estar saludable y en buena forma física, relajarse, aumentar y mantener el entorno social, aumentar y mantener la confianza en sí mismo, aumentar la autoestima, salir de casa, prescripción médica, otros), siendo utilizada una escala tipo Likert de 5 puntos, cuyo significado se explica a continuación.

Respecto a las barreras y los facilitadores, se debe puntuar la afirmación como barrera muy importante (1), barrera (2), ni barrera ni facilitador (3), facilitador (4) y facilitador muy importante (5).

En los motivos para la práctica, se debe puntuar la afirmación en función del nivel de importancia respecto a los diferentes motivos como no importante (1), un poco importante (2), neutral (3), importante (4) y muy importante (5).

\section{Variables dependientes}

Las variables dependientes utilizadas en el presente trabajo se han agrupado en dos grandes grupos:

Barreras y facilitadores: indica si los factores presentados suponen una barrera o un facilitador hacia la participación en actividades físicas y deportivas. Se han analizado en función de las respuestas de los participantes en función de 4 factores diferentes. Cada cuestión ha sido considerada por separado y también agrupadas en medias por factores:

Desplazamiento: compuesto por 5 ítems en los que se preguntaba sobre el nivel de dificultad para desplazarte al lugar de entrenamiento.

Tiempo: compuesto por 4 ítems en los que se preguntaba sobre el nivel de dificultad respecto al tiempo.

Instalaciones: compuesto por 7 ítems en los que se preguntaba sobre el nivel de dificultad respecto a las instalaciones y el material.

Apoyo: compuesto por 7 ítems en los que se pregunta sobre el nivel de dificultad respecto al apoyo proporcionado por otros.

Motivos para la práctica: compuesto por 8 ítems en los que los participantes deben indicar el nivel de importancia que le otorgan a los motivos de participación que se presentan.

\section{Variables independientes}

Sexo: se diferencias entre hombres y mujeres.

Carga de entrenamiento: se diferencia entre los participantes que entrenaban más de 3 horas a la semana y los que entrenaban 3 horas o menos a la semana.

Ocupación: se diferencia entre los participantes que trabajaban, los que estudiaban y otros (compuesto por los participantes que estaban jubilados, desempleados y que están en casa o en una institución). 


\section{Procedimiento}

El protocolo utilizado en este estudio está de acuerdo con las exigencias de la Declaración de Helsinki (2000). Cada participante recibió una invitación a participar, explicando los objetivos del mismo y obteniendo el consentimiento informado de los participantes.

Como se ha indicado en el apartado instrumentos, se ha utilizado un cuestionario administrado en forma de entrevista. Los participantes debían responder a las preguntas relacionadas con los diferentes factores del cuestionario. Para ello recibieron ayuda del investigador principal y de sus entrenadores. El tiempo aproximado empleado para responder el cuestionario fue de 20 minutos. La recogida de los datos se realizó en el lugar de entrenamiento, antes de comenzar el mismo y asegurando las condiciones de aplicación en un lugar retirado y tranquilo.

\section{Análisis estadístico}

Se ha utilizado la estadística descriptiva (frecuencias, medias, desviación típica, valores máximos y mínimos). Para el análisis inferencial se han utilizado pruebas no paramétricas ( $U$ de Mann-Whitney, Kruskal-Wallis, correlaciones de Spearman). El análisis post-hoc se ha realizado a partir de la utilización de las pruebas estadísticas Turkey HSD y Games-Howell. El nivel de significación fue $p \leq 0,05$. Todos los datos fueron analizados mediante el Statistical Package for Social Sciences (versión 22.0 para Windows, SPSS Inc, Chicago, IL, USA), y el nivel de significación fue establecido en $\mathrm{p}<0.05$.

\section{Resultados}

A continuación se presentan los resultados obtenidos a partir del análisis de datos realizado. La Tabla 1 muestra las puntuaciones medias globales en cada uno de los factores para el total de los participantes, tanto en cada una de las preguntas que construían el factor como en la media de dicho factor. 
Tabla 1. Valores Medios en los Factores Analizados

\begin{tabular}{lcccc}
\hline Factor & M & DT & Mínimo & Máximo \\
\hline Desplazamiento & 3.80 & .62 & 2.80 & 5 \\
Horario & 3.95 & .74 & 3 & 5 \\
Transporte adaptado & 3.71 & .72 & 3 & 5 \\
Tiempo & 3.68 & .89 & 2 & 5 \\
Coste & 3.81 & .81 & 2 & 5 \\
N $^{\circ}$ transportes & 3.81 & .87 & 2 & 5 \\
Tiempo & 3.86 & .86 & 2.50 & 5 \\
Llegar al entrenamiento & 3.83 & 1.19 & 1 & 5 \\
Prepararte para entrenar & 3.80 & .83 & 3 & 5 \\
Ir a la zona de entrenamiento & 4.04 & .93 & 1 & 5 \\
Próximo compromiso & 3.74 & 1.13 & 1 & 5 \\
Instalaciones & 4.05 & .95 & 1 & 5 \\
Acceso instalaciones & 4.04 & .98 & 1 & 5 \\
Acceso vestuarios & 4.00 & 1.09 & 1 & 5 \\
Uso servicio & 4.00 & 1.00 & 1 & 5 \\
Cambiarse de ropa & 4.04 & 1.02 & 1 & 5 \\
Ducharse & 3.83 & 1.07 & 1 & 5 \\
Acceso área deportiva & 4.17 & 1.03 & 1 & 5 \\
Materiales adaptados & 4.26 & .96 & 1 & 5 \\
Apoyo & 4.26 & .66 & 2.71 & 5 \\
Amigos & 4.18 & 1.07 & 1 & 5 \\
Maestros/instructores & 4.52 & .57 & 3 & 5 \\
Compañeros de entrenamiento & 4.05 & 1.11 & 1 & 5 \\
Personal instalaciones & 4.33 & .74 & 2 & 5 \\
Otros usuarios & 4.05 & .98 & 1 & 5 \\
Ayudantes & 4.43 & .57 & 3 & 5 \\
Padres o tutores & 4.26 & .90 & 1 & 5 \\
\hline
\end{tabular}

Los resultados indican que las principales barreras percibidas por los deportistas con DI son: "el tiempo que tardas para desplazarse a su lugar de entrenamiento" (3.68), "la existencia de transporte adaptado" (3.71) y "el tiempo que tardas en llegar a tu próximo compromiso" (3.74).

En cuanto a los principales facilitadores, los resultados muestran que son: "el apoyo de los maestros/instructores" (4.52), "el apoyo de los ayudantes de los deportistas" (4.43) y "el apoyo del personal de las instalaciones" (4.33).

La Tabla 2 muestra las puntuaciones medias en cada uno de los factores analizados en función del sexo de los participantes (hombres vs. mujeres) y de la carga de entrenamiento semanal de los participantes ( $\leq 3$ horas vs. $>3$ horas). 
Tabla 2. Valores medios de los factores y de las barreras y facilitadores analizados en función del sexo y de la carga de entrenamiento

\begin{tabular}{|c|c|c|c|c|c|c|c|c|}
\hline \multirow[b]{3}{*}{ Factor } & \multicolumn{4}{|c|}{ Sexo } & \multicolumn{4}{|c|}{ Carga entrenamiento } \\
\hline & \multicolumn{2}{|c|}{ Hombres } & \multicolumn{2}{|c|}{ Mujeres } & \multicolumn{2}{|c|}{$\leq 3$ horas } & \multicolumn{2}{|c|}{$>3$ horas } \\
\hline & $\mathrm{M}$ & DT & $\mathrm{M}$ & DT & $\mathrm{M}$ & DT & $\mathrm{M}$ & DT \\
\hline Desplazamiento** & 3.63 & .57 & 4.10 & .63 & 3.99 & .45 & 3.48 & .77 \\
\hline Horario & 3.77 & .60 & 4.25 & .89 & 4.15 & .69 & 3.63 & .74 \\
\hline Transporte adaptado & 3.54 & .52 & 4.00 & .93 & 3.77 & .73 & 3.63 & .64 \\
\hline Tiempo & 3.43 & .94 & 4.13 & .64 & 3.93 & .62 & 3.25 & 1.17 \\
\hline Coste & 3.77 & .83 & 3.88 & .84 & 4.00 & .71 & 3.50 & .93 \\
\hline $\mathrm{N}^{\mathrm{o}}$ transportes & 3.54 & .78 & 4.25 & .89 & 4.08 & .76 & 3.38 & .92 \\
\hline Tiempo & 3.71 & .51 & 4.10 & 1.22 & 3.96 & .91 & 3.72 & .80 \\
\hline Llegar al entrenamiento & 3.64 & 1.08 & 4.11 & 1.36 & 3.93 & 1.21 & 3.67 & 1.23 \\
\hline Prepararte para entrenar & 3.68 & .75 & 4.17 & .98 & 4.00 & .85 & 3.50 & .76 \\
\hline Ir a la zona de entrenamiento & 4.00 & .56 & 4.11 & 1.36 & 4.07 & 1.07 & 4.00 & .71 \\
\hline Próximo compromiso & 3.57 & .94 & 4.00 & 1.42 & 3.86 & 1.17 & 3.56 & 1.13 \\
\hline Instalaciones & 3.94 & .53 & 4.22 & 1.39 & 4.06 & 1.06 & 4.03 & .80 \\
\hline Acceso instalaciones & 3.93 & .62 & 4.22 & 1.39 & 4.07 & 1.07 & 4.00 & .87 \\
\hline Acceso vestuarios & 3.86 & .86 & 4.22 & 1.39 & 4.07 & 1.14 & 3.89 & 1.05 \\
\hline Uso servicio & 3.86 & .66 & 4.22 & 1.39 & 4.00 & 1.11 & 4.00 & .87 \\
\hline Cambiarse de ropa & 3.93 & .73 & 4.22 & 1.39 & 4.07 & 1.14 & 4.00 & .87 \\
\hline Ducharse* & 3.57 & .76 & 4.22 & 1.39 & 3.86 & 1.10 & 3.78 & 1.09 \\
\hline Acceso área deportiva & 4.14 & .77 & 4.22 & 1.42 & 4.14 & 1.17 & 4.22 & .83 \\
\hline Materiales adaptados & 4.29 & .61 & 4.22 & 1.39 & 4.21 & 1.12 & 4.33 & .71 \\
\hline Apoyo & 4.37 & .37 & 4.09 & .95 & 4.23 & .80 & 4.31 & .36 \\
\hline Amigos & 4.50 & .65 & 3.69 & 1.42 & 3.94 & 1.27 & 4.56 & .53 \\
\hline Maestros/instructores & 4.50 & .52 & 4.56 & .68 & 4.61 & .63 & 4.39 & .49 \\
\hline Compañeros de entrenamiento & 4.22 & .80 & 3.79 & 1.48 & 4.01 & 1.24 & 4.12 & .93 \\
\hline Personal instalaciones & 4.38 & .43 & 4.26 & 1.09 & 4.33 & .91 & 4.33 & .41 \\
\hline Otros usuarios & 4.15 & .66 & 3.89 & 1.37 & 4.07 & 1.21 & 4.01 & .50 \\
\hline Ayudantes & 4.39 & .49 & 4.49 & .71 & 4.46 & .63 & 4.38 & .48 \\
\hline Padres o tutores & 4.47 & .49 & 3.95 & 1.28 & 4.20 & 1.10 & 4.36 & .49 \\
\hline
\end{tabular}

* indica diferencias significativas entre los grupos por sexo para $p \leq 05$.

** indica diferencias significativas entre los grupos por carga de entrenamiento para $p \leq 05$.

Los principales facilitadores para los hombres son "el apoyo de los amigos" (4.50) y el "apoyo de los maestros/instructores" (4.50) mientras que para las mujeres son "el apoyo de los maestros/instructores" (4.56) y "el apoyo de los ayudantes" (4.49). La comparación de las barreras y los facilitadores en función del sexo mostró que existían diferencias estadísticamente significativas en relación a "ducharse en el vestuario" $(U=32.00, p=.041)$. Los hombres consideraban este factor como una barrera al compararlos con las mujeres. El resto de los factores analizados no mostraron diferencias estadísticamente significativas en función del sexo.

Los principales facilitadores en función de la carga de entrenamiento para el grupo con una carga de entrenamiento inferior o igual a 3 horas semanales son "el apoyo de los ayudantes" (4.33) y el "apoyo del personal de las instalaciones" (4.33) mientras que para el 
grupo con una carga de entrenamiento superior a 3 horas semanales son "el apoyo de los amigos" (4.56) y "el apoyo de los maestros/instructores" (4.39). La comparación de las barreras $\mathrm{y}$ los facilitadores en función de la carga de entrenamiento ha revelado diferencias significativas solo para el valor medio del factor "desplazamiento" $(U=27,50, p=0,050)$. El grupo con una carga de entrenamiento semanal superior ( $>3$ horas) obtiene un valor significativamente más bajo para el factor "desplazamiento" que el grupo con una carga de entrenamiento inferior. El resto de los factores analizados no mostraron diferencias estadísticamente significativas en función de la carga de entrenamiento.

La Tabla 3 muestra las puntuaciones medias en cada uno de los factores analizados en función de la ocupación diaria de los participantes, creando tres grupos de análisis (trabajadores, estudiantes y otros).

Tabla 3. Valores medios de los factores y las preguntas analizados en función de la ocupación diaria

\begin{tabular}{lcccccc} 
& \multicolumn{7}{c}{ Ocupación diaria } \\
\cline { 2 - 6 } Factor & \multicolumn{2}{c}{ Trabajadores } & \multicolumn{2}{c}{ Estudiantes } & \multicolumn{2}{c}{ Otros } \\
\cline { 2 - 7 } & $\mathrm{M}$ & $\mathrm{DT}$ & $\mathrm{M}$ & $\mathrm{DT}$ & $\mathrm{M}$ & $\mathrm{DT}$ \\
\hline Desplazamiento & 3.86 & .69 & 3.68 & .50 & 3.80 & .67 \\
Horario & 3.90 & .74 & 3.80 & .84 & 4.17 & .75 \\
Transporte adaptado & 3.80 & .80 & 3.60 & .89 & 3.67 & .52 \\
Tiempo & 3.64 & 1.03 & 3.60 & .55 & 3.83 & .98 \\
Coste & 4.00 & .67 & 3.60 & .89 & 3.67 & 1.03 \\
$\mathrm{~N}^{\text {o transportes }}$ & 3.90 & .88 & 3.80 & .84 & 3.67 & 1.03 \\
Tiempo & 4.00 & .57 & 3.50 & 1.50 & 3.92 & .71 \\
Llegar al entrenamiento & 3.91 & 1.14 & 3.40 & 1.67 & 4.00 & 1.00 \\
Prepararte para entrenar & 4.10 & .74 & 3.80 & 1.10 & 3.20 & .45 \\
Ir a la zona de entrenamiento & 4.18 & .60 & 3.40 & 1.67 & 4.29 & .49 \\
Próximo compromiso & 3.82 & .98 & 3.40 & 1.67 & 3.86 & 1.07 \\
Instalaciones & 4.13 & .65 & 3.40 & 1.67 & 4.38 & .47 \\
Acceso instalaciones & 4.09 & .70 & 3.40 & 1.67 & 4.43 & .54 \\
Acceso vestuarios & 4.00 & 1.00 & 3.40 & 1.67 & 4.43 & 1.36 \\
Uso servicio & 4.00 & .78 & 3.40 & 1.67 & 4.43 & .54 \\
Cambiarse de ropa & 4.18 & .75 & 3.40 & 1.67 & 4.29 & .76 \\
Ducharse & 3.82 & .98 & 3.40 & 1.67 & 4.14 & .69 \\
Acceso área deportiva & 4.36 & .67 & 3.40 & 1.67 & 4.43 & .79 \\
Materiales adaptados & 4.45 & .52 & 3.40 & 1.53 & 4.57 & .54 \\
Apoyo & 4.56 & .40 & 3.79 & .91 & 4.13 & .62 \\
Amigos & 4.64 & .67 & 3.44 & 1.54 & 4.00 & 1.00 \\
Maestros/instructores & 4.73 & .47 & 4.30 & .84 & 4.36 & .48 \\
Compañeros de entrenamiento & 4.28 & .90 & 3.41 & 1.52 & 4.15 & 1.07 \\
Personal instalaciones & 4.61 & .47 & 4.33 & .82 & 3.90 & .92 \\
Otros usuarios & 4.37 & .67 & 3.42 & 1.53 & 4.00 & .82 \\
Ayudantes & 4.68 & .47 & 4.29 & .83 & 4.14 & .38 \\
Padres o tutores* & 4.66 & .48 & 3.31 & 1.39 & 4.32 & .47 \\
\hline
\end{tabular}

* indica diferencias significativas entre los grupos para $p \leq 05$. 
Los principales facilitadores mostrados por los participantes en función de la ocupación muestran que el grupo de trabajadores presenta como principales facilitadores "el apoyo de los maestros/instructores" (4.73) y "el apoyo de los ayudantes" (4.68), para el grupo de estudiantes los principales facilitadores son "el apoyo del personal de las instalaciones" (4.33) y "el apoyo de maestros/instructores" (4.30), finalmente para el grupo otros el principal facilitador es "la existencia de materiales adaptados" (4.57) seguido de varios aspectos relacionados con las instalaciones. La comparación de las barreras y los facilitadores en función de la ocupación de los participantes ha mostrado diferencias estadísticamente significativas. El grupo de trabajadores obtuvo valores significativamente más altos en el factor "el apoyo de los padres" al compararlo con el grupo de los estudiantes $(U=8.50, p=.023)$. El resto de factores analizados no arrojaron diferencias estadísticamente significativas para ninguno de los grupos analizados en función de la ocupación.

La Tabla 4 presenta los valores medios de los participantes en cuanto a los motivos para la práctica de actividad física y deportiva.

Tabla 4. Valores medios de los motivos para la práctica de actividades fisicas y deportivas

\begin{tabular}{lcccc}
\hline Motivos & M & DT & Mínimo & Máximo \\
\hline Estar saludable & 4.73 & .70 & 2 & 5 \\
Relajarse & 3.95 & 1.43 & 1 & 5 \\
Aumentar y mantener mi entorno social & 4.64 & .90 & 1 & 5 \\
Aumentar y mantener la confianza en mí mismo & 4.68 & .89 & 1 & 5 \\
Aumentar mi autoestima & 4.41 & 1.22 & 1 & 5 \\
Salir de casa & 4.41 & 1.01 & 1 & 5 \\
Prescripción médica & 4.09 & 1.19 & 1 & 5 \\
\hline
\end{tabular}

Los resultados muestran que los principales motivos para practicar actividad física y deportiva mostrados por los participantes son: "estar saludable" (4.73), "aumentar y mantener la confianza en mí mismo" (4.68) y "aumentar y mantener mi entorno social" (4.64). Al relacionar los resultados de cada uno de los motivos, se ha constatado la existencia de asociaciones positivas entre algunos de los motivos. "Salir de casa y aumentar la autoestima" están muy asociados con "aumentar y mantener mi entorno social" $\left(r_{s}=.462, \mathrm{p}=.026\right.$ y $r_{s}=.627$, $\mathrm{p}=.001$, respectivamente). Otra de las asociaciones positivas encontradas fue "aumentar mi autoestima" y "aumentar y mantener la confianza en mí mismo" $\left(r_{s}=.538, \mathrm{p}=.008\right)$.

Adicionalmente se han realizado comparaciones en función de las variables independientes (sexo, carga de entrenamiento y ocupación), con el objetivo de encontrar diferencias en cuanto a los motivos de participación en actividades físicas y deportivas. Las pruebas estadísticas no han mostrado diferencias significativas para ninguna de las agrupaciones analizadas.

\section{Discusión}

El objetivo de este estudio fue describir las barreras y los facilitadores percibidos por las personas con DI de cara a su participación en actividades físicas y deportivas. Adicionalmente se han explorado también los motivos para la práctica en esta población. Los resultados han 
sido comparados en función del sexo, la carga de entrenamiento de los participantes y la ocupación de los participantes.

Los resultados nos indican que el tiempo es la barrera más importante a la que se enfrentan las personas con DI de la presente investigación a la hora de practicar deporte, estos resultados aparecen tanto en el global de los resultados como en la agrupación de la carga de entrenamiento. Como parece lógico, a medida que son más los días de entrenamiento y la carga semanal de entrenamiento, la necesidad de desplazarse al lugar de entrenamiento comienza a ser una barrera importante. Es posible que la escasez de oferta deportiva para este colectivo haga que las personas con DI que deseen practicar deporte tengan que desplazarse para practicarlo, al carecer de oferta deportiva cerca de su domicilio. Los estudios previos en el conjunto de las personas con discapacidad no habían detectado el tiempo como una barrera, sin embargo si habían encontrado barreras muy relacionadas con el desplazamiento, como el transporte y la ausencia de instalaciones (Jaarsma, Dijkstra, Geertzen y Dekker, 2014), lo que sin duda influye en el tiempo empleado para llegar al lugar de entrenamiento.

Los resultados en función del sexo muestran que ducharse en el vestuario es una barrera importante para los hombres en comparación de las mujeres. Previamente se habían encontrado diferencias en función del sexo en la búsqueda de barreras y facilitadores para la práctica de actividad física y deporte. Por ejemplo, Yoh, Mohr y Gordon (2008) encontraron que el sexo era una barrera para los estudiantes universitarios con discapacidad física en la utilización de las instalaciones de ocio de la universidad, sin embargo, a diferencia del presente trabajo, en el trabajo de Yoh et al. (2008), la barrera era ser mujer. También en el trabajo de Ajuwon et al. (2008) mostraron esta tendencia, encontraron que ser hombre aparecía como un facilitador para la práctica de actividad física y deportiva recreativa en personas con discapacidad visual en Nigeria. En el trabajo actual los participantes masculinos encuentran un problema en ducharse en las instalaciones deportivas, sin embargo en virtud de los datos obtenidos no es posible determinar si la dificultad tiene que ver con las instalaciones o con algunas de sus características personales, por tanto es necesario más investigación al respecto con el objeto de obtener una información más completa sobre este aspecto.

En cuanto a los facilitadores, los aspectos que más ayudan a la participación de personas con DI en actividades físicas y deportivas tienen que ver con el apoyo proporcionado por otros. Los resultados globales muestran que el apoyo de las personas cercanas al entorno deportivo (maestros/instructores, ayudantes y personal de la instalación) es un facilitador muy importante, además al comparar los resultados en función de la carga de entrenamiento, el apoyo de los padres o tutores también es muy relevante. Estos resultados están de acuerdo con estudios previos en el ámbito del deporte para personas con discapacidad, en los que tradicionalmente se ha identificado el soporte familiar y de los profesionales como un facilitador muy importante para la práctica deportiva (Greguol et al., 2015; Jaarsma, Geertzen et al., 2014; Perkins et al., 2013). Por ejemplo, en el trabajo de Jaarsma, Geertzen et al. (2014) el soporte social fue uno de los facilitadores mostrados por los deportistas paralímpicos holandeses a la hora de iniciarse en el deporte. A partir de estos resultados y de acuerdo con Perkins et al. (2013) podemos decir que es necesario incluir al entorno del deportista (especialmente a los padres) a la hora de diseñar e implementar programas con el objetivo de incrementar la participación de las personas con discapacidad (con DI si nos referimos específicamente a la población del presente trabajo) en actividades físicas y deportivas. 
En la población objeto de estudio es particularmente importante la necesidad de practicar actividad física y deportiva con el objetivo de estar saludable. Se ha demostrado que las personas con DI son especialmente vulnerables a los problemas de salud relacionados con el sedentarismo, de hecho la obesidad ha sido identificado como el problema de salud más importante en dicho colectivo (De Winter, Magilsen, Van Alfer, Penning y Evenhuis, 2009), lo que ha llevado a la necesidad de implementar programas destinados a la mejora de la salud en personas con DI a través de programas de actividad física y deporte regular (Ordoñez et al., 2013). Parece que esta idea está arraiga en la población de estudio del presente trabajo, ya que su principal motivo para practicar actividad física y deportiva es precisamente estar saludable, seguido de aumentar la confianza en sí mismos y aumentar su entorno social.

Además se ha comprobado que existe una relación entre la intención de aumentar y mantener el entorno social, con la necesidad de salir de casa y de aumentar la autoestima. Existe una relación entre aumentar y mantener la autoestima y aumentar y mantener la confianza en sí mismo. Parece que la necesidad de mantener un entorno social hace que los deportistas tengan que salir de casa y eso les produce una mejora de su autoestima y de su confianza en sí mismos. Este hallazgo está de acuerdo con la idea de que la práctica de actividad física y deportiva en las personas con discapacidad es una poderosa herramienta para favorecer su socialización, conviniendo con estudios previos como el de Crawford et al. (2008).

\section{Conclusiones}

Teniendo en cuenta los objetivos previstos en la investigación y las limitaciones, relacionadas con la escasez de la muestra, podemos extraer las siguientes conclusiones:

Las principales barreras detectadas están relacionadas con el tiempo, por lo que se propone crear una mayor oferta deportiva que permita a las personas con DI tener acceso a posibilidades de práctica deportiva más cercanas.

Los principales facilitadores se han relacionado con el apoyo del entorno del deportista, por lo que se propone que para implementar y mantener una oferta deportiva acorde a la población estudiada se debería contar con la opinión y participación del entorno familiar y deportivo de las personas con DI.

Los motivos fundamentales de práctica de actividad física y deportiva están relacionados con la salud y los factores sociales (salir de casa, mejora de autoestima y aumentar y mantener el entorno social), por lo que fomentar la práctica deportiva es este colectivo es necesario para mejorar su calidad de vida.

Estos hallazgos deben ser tenidos en cuenta por los profesionales de la actividad física, la Educación Física y el deporte para personas con DI con el objetivo de la mejora del ámbito profesional, para implementar ofertas adecuadas a las necesidades y características específicas de esta población. Además suponen un punto de partida para realizar investigaciones y de esta manera ampliar el campo de conocimiento de la actividad física y el deporte para personas con DI.

Como propuestas de investigación futuras, se sugiere la utilización de una metodología mixta (cuantitativa y cualitativa) con el objetivo de obtener una comprensión más profunda de las barreras, facilitadores y motivos de la práctica deportiva en las personas con DI, debido a su importancia tanto desde el punto de vista teórico como desde el punto de vista de las implicaciones prácticas. 


\section{Referencias bibliográficas}

Abellán, J. y Sáez-Gallego, N. M. (2014). Justificación de las pruebas motrices en el deporte para personas con discapacidad intelectual. Revista Iberoamericana de Psicología del Ejercicio y el Deporte, 9(1), 143-153.

Ajuwon, P. M., Kelly, S. M. y Wolffe, K. E. (2015). The recreation and leisure pursuits of employed adults with visual impairments in Nigeria: Part 2. Journal of Visual Impairment y Blindness (Online), 109(1), 31.

Badia, M., Orgaz, B. M., Verdugo, M. A., Ullán, A. M. y Martínez, M. M. (2011). Personal factors and perceived barriers to participation in leisure activities for young and adults with developmental disabilities. Research in Developmental Disabilities, 32(6), 20552063. https://doi.org/10.1016/j.ridd.2011.08.007

Block, M. E. y Obrusnikova, I. (2007). Inclusion in physical education: A review of the literature from 1995-2005. Adapted physical activity quarterly, 24(2), 103-124. https://doi.org/10.1123/apaq.24.2.103

Brian, A. y Haegele, J. A. (2014). Including students with visual impairments: Softball. Journal of Physical Education, Recreation and Dance, 85(3), 39-45. https://doi.org/10.1080/07303084.2014.875808

Bult, M. K., Verschuren, O., Jongmans, M. J., Lindeman, E. y Ketelaar, M. (2011). What influences participation in leisure activities of children and youth with physical disabilities? A systematic review. Research in developmental disabilities, 32(5), 1521 1529. https://doi.org/10.1016/j.ridd.2011.01.045

Crawford, A., Hollingsworth, H. H., Morgan, K. y Gray, D. B. (2008). People with mobility impairments: Physical activity and quality of participation. Disability and health journal, 1(1), 7-13. https://doi.org/10.1016/j.dhjo.2007.11.004

Damas, R., Januário, N., Rosado, A. y Pereira, L. (2016). Barriers and facilitators of sports participation for Portuguese wheelchair handball players. European Journal of Adapted Physical Activity, 9 (Suppl.), 116.

De Winter, C. F., Magilsen, K. W., Van Alfen, J. C., Penning, C. y Evenhuis, H. M. (2009). Prevalence of cardiovascular risk factors in older people with intellectual disability. American journal on intellectual and developmental disabilities, 114(6), 427-436. https://doi.org/10.1352/1944-7558-114.6.427

Greguol, M., Gobbi, E. y Carraro, A. (2015). Physical activity practice among children and adolescents with visual impairment-influence of parental support and perceived barriers. Disability and rehabilitation, 37(4), 327-330. https://doi.org/10.3109/09638288.2014.918194

Haegele, J. A. y Porretta, D. (2015). Physical activity and school-age individuals with visual impairments: A literature review. Adapted Physical Activity Quarterly, 32(1), 68-82. https://doi.org/10.1123/apaq.2013-0110

Jaarsma, E. (2014). Sports participation and physical disabilities: Taking the hurdle?! Tesis doctoral publicada. University of Groningen. Recuperada de http://hdl.handle.net/11370/4ef9605d-3f97-4d55-94b2-5ca3b9ccd5b5 (13/03/2017). 
Jaarsma, E. A., Dijkstra, P. U., Geertzen, J. H. B. y Dekker, R. (2014). Barriers to and facilitators of sports participation for people with physical disabilities: A systematic review. Scandinavian journal of medicine \& science in sports, 24(6), 871-881. https://doi.org/10.1111/sms.12218

Jaarsma, E. A., Geertzen, J. H., Jong, R. D., Dijkstra, P. U. y Dekker, R. (2014). Barriers and facilitators of sports in Dutch Paralympic athletes: An explorative study. Scandinavian journal of medicine y science in sports, 24(5), 830-836. https://doi.org/10.1111/sms.12071

Januário, N., Colaço, C., Rosado, A., Ferreira, V., \& Gil, R. (2012). Students motivations for sport involvement: The effect of age, gender and school level. Motricidade, 8(4), 38-51.

Kirchner, C. E., Gerber, E. G. y Smith, B. C. (2008). Designed to deter: community barriers to physical activity for people with visual or motor impairments. American journal of preventive medicine, 34(4), 349-352. https://doi.org/10.1016/j.amepre.2008.01.005

Lieberman, L. J., Robinson, B. L. y Rollheiser, H. (2006). Youth with visual impairments: Experiences in general physical education. RE: view: Rehabilitation Education for Blindness and Visual Impairment, 38(1), 35-48. https://doi.org/10.3200/REVU.38.1.35-48.

Mendoza, N. (2009). Propuestas prácticas de Educación Física inclusiva para la etapa de secundaria. Barcelona: INDE.

Ordoñez, F. J., Rosety, M. A., Camacho, A., Rosety, I., Diaz, A. J., Fornieles, G., ... у Rosety-Rodriguez, M. (2014). Aerobic training improved low-grade inflammation in obese women with intellectual disability. Journal of Intellectual Disability Research, 58(6), 583-590. https://doi.org/10.1111/jir.12056.

Organización Mundial de la Salud (OMS) (2011). International Classification of Functioning, Disability and Health (ICF).

Perkins, K., Columna, L., Lieberman, L. y Bailey, J. (2013). Parents' perceptions of physical activity for their children with visual impairments. Journal of Visual Impairment $y$ Blindness (Online), 107(2), 131.

Robertson, J. y Emerson, E. (2010). Participation in sports by people with Intellectual Disabilities in England: A brief report. Journal of Applied Research in Intellectual Disabilities, 23(6), 616-622. https://doi.org/10.1111/j.1468-3148.2009.00540.x.

Torralba, M. A., Braz, M. y Rubio, M. J. (2017). Motivos para la práctica deportiva de atletas paralímpicos españoles. Revista de Psicología del Deporte, 26 (1), 49-60.

Yazdani, S., Yee, C. T. y Chung, P. J (2013). Factors predicting physical activity among children with special needs. Preventing chronic disease, 10, https://doi.org/10.5888/pcd10.120283.

Yoh, T., Mohr, M. y Gordon, B. (2008). Assessing satisfaction with campus recreation facilities among college students with physical disabilities. Recreational Sports Journal, 32(2), 106-113. https://doi.org/10.1123/rsj.32.2.106. 